\title{
Stratégie de pâturage d'une population d'éléphants en saison sèche : \\ le cas du parc de Zakouma au Tchad
}

De Pierre Poilecot et en son hommage ${ }^{1}$ *

Parachevé par :

Slim SAÏDI ${ }^{1}$ et Philippe DAGET ${ }^{2}$

1 Cirad

Umr Tetis

Campus international

de Baillarguet

34398 Montpellier Cedex 5

France

2 Cirad-Cnrs

Umr Selmet

Campus international

de Baillarguet

34398 Montpellier Cedex 5

France

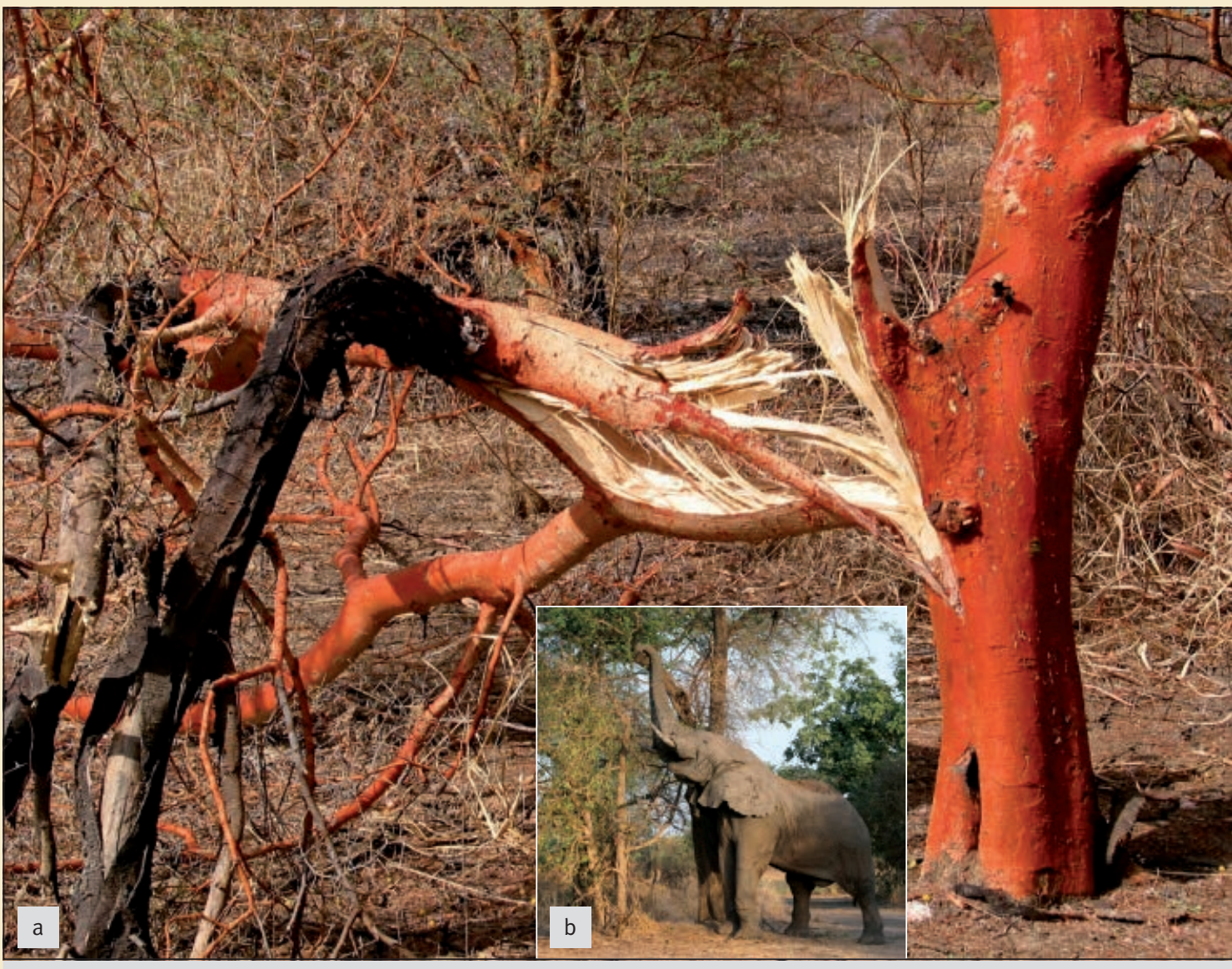

Acacia seyal cassé par un éléphant (a). Éléphant au pâturage sur un Balanites aegyptiaca (b). Photo P. Poilecot.

* Pierre Poilecot, décédé en juin 2012, a longuement séjourné au parc de Zakouma où il a mené des études de botanique, d'écologie et d'éthologie. Il a toujours fait connaître ses observations et ses interprétations à ses collègues et analysé avec eux les relations entre les caractéristiques physiques du milieu, la végétation, spécialement la végétation ligneuse, et le comportement des éléphants. Pour le présent article ont été exploitées les notes qu’il a laissées. 


\section{RÉSUMÉ}

\section{STRATÉGIE DE PÂTURAGE D’UNE POPULATION D’ÉLÉPHANTS EN SAISON SÈCHE : LE CAS DU PARC DE ZAKOUMA AU TCHAD}

Les peuplements riches en Acacia seyal des savanes du Sud-Est du Tchad constituent en saison sèche l'une des principales ressources alimentaires pour la population d'éléphants du parc national de Zakouma. Sous forme de mosaïques plus au moins denses, ces formations arborées constituent un complément de nutrition indispensable pour l'équilibre alimentaire de ces grands herbivores. Dans cette note, il ne s'agit pas d'estimer le potentiel de ces savanes en pâturage aérien disponible vis-à-vis des éléphants du parc, mais d'identifier grâce à une approche spatiale les surfaces qui s'avèrent déterminantes ou marginales pour leur alimentation. Ainsi l'imagerie satellitaire a permis d'établir des valeurs quantitatives et spatiales de cette ressource végétale dont l'utilisation n'apparaît pas être liée à la qualité alimentaire ou à l'appétence, mais le résultat de divers facteurs physiques et édaphiques concomitants que sont la permanence du réseau hydrique et le seuil de densité supérieur à 400 arbres à l'hectare limitant l'accès des troupeaux en saison sèche.

Mots-clés : savane, éléphants, Acacia seyal, image satellite, Zakouma, Tchad.

\section{SUMMARY}

\section{DRY-SEASON GRAZING STRATEGY OF AN ELEPHANT POPULATION IN ZAKOUMA PARK IN CHAD}

During the dry season, Acacia seyal stands in the savannah areas of southeastern Chad are one of the main food sources for the elephant population of Zakouma National Park. Forming a more or less dense mosaic, this tree savannah is an essential additional source of nutrition for these huge herbivores. This note does not set out to estimate the aerial grazing potential of the savannah for the elephants in the park, but to identify, through remote sensing, the areas that are either essential or marginal in terms of their food needs. Satellite imagery was used to establish quantitative and spatial values for these vegetation resources, whose use does not appear to be related to food quality or palatability, but to result from several concomitant physical and edaphic factors, i.e. a permanent water network and the fact that tree densities of more than 400 trees per hectare limit access for the elephants to these dry-season food resources.

Keywords: savannah, elephants, Acacia seyal, satellite imagery, Zakouma, Chad.

\section{RESUMEN}

\section{ESTRATEGIA DE PASTOREO DE UNA POBLACIÓN DE ELEFANTES EN LA ESTACIÓN SECA: EL CASO DEL PARQUE DE ZAKOUMA EN CHAD}

Los rodales con abundancia de Acacia seyal de las sabanas del sudeste del Chad son uno de los principales recursos alimenticios durante la estación seca para la población de elefantes del Parque Nacional de Zakouma. Estas formaciones arbóreas, según mosaicos más o menos densos, constituyen un complemento nutricional indispensable para el equilibrio alimentario de estos grandes herbívoros. En esta nota no se trata de estimar el potencial de los pastos aéreos de estas sabanas, sino de identificar mediante un enfoque espacial las áreas que resultan determinantes o marginales para su alimentación. Es así como gracias a las imágenes satelitales, se lograron determinar los valores cuantitativos y espaciales de este recurso vegetal cuya utilización no parece que esté relacionada con la calidad alimentaria o la apetencia, sino que es el resultado de diferentes factores físicos y edáficos concomitantes. Dichos factores son la permanencia de la red hídrica y el hecho que una densidad superiora 400 árboles por hectárea limita el acceso de las manadas en la estación seca.

Palabras clave: sabana, elefantes, Acacia seyal, imagen satelital, Zakouma, Chad. 


\section{Introduction}

Les peuplements riches en Acacia seyal (Del.) constituent des formations végétales denses au sein des plaines périodiquement inondées de la zone sahélo-soudanienne. Acacia seyal occupe au Tchad une vaste aire de distribution, atteignant $16^{\circ}$ de latitude Nord, et qui s'étend jusqu'à la limite du massif de l'Ennedi, à la frontière du Soudan. Cette espèce s'adapte bien aux inondations temporaires et colonise les vertisols ainsi que les sols lourds et battants dont la teneur en argile noire atteint des taux relativement élevés (PIAS, POISOT, 1964).

En saison sèche, les graminées mûres desséchées sont extrêmement pauvres en protéines, phosphore et carotène et ne peuvent plus couvrir les besoins d'entretien des troupeaux d'éléphants du parc. De ce fait, la consommation des ligneux en cette période de l'année devient un complément indispensable à l'équilibre alimentaire de ces grands herbivores (4 à $7 \%$ du poids vif ; POILECOT et al., 2007).

Ces vastes étendues d'Acacia seyal, situées à l'Est du parc de Zakouma, représentent une ressource alimentaire mise à profit par les éléphants. Certains peuplements sont fortement parcourus et pâturés, alors que d'autres ne le sont guère (MAIRE, 2000 ; CALENGE et al., 2002). C'est dans le contexte de besoins d'aménagement et de conservation qu'un projet ${ }^{1}$ de gestion des ressources a mis en œuvre, dès 2003, un programme d'inventaire de la végétation à l'échelle du parc, afin d'évaluer l'impact du pâturage aérien des éléphants sur les formations de savanes à Acacia seyal et à Combretaceae afin d'identifier les surfaces qui s'avèrent déterminantes ou marginales pour leur alimentation.

Le comportement alimentaire des éléphants est peu documenté scientifiquement. La présente démarche contribue à mieux appréhender cette activité au cours des phases de pâturage, et cela grâce à une approche floristique et spatiale, que permet l'utilisation de l'indice perpendiculaire de végétation $(\mathrm{PVI})$.

\section{Zone d'étude}

Le parc de Zakouma couvre une surface de $3000 \mathrm{~km}^{2}$. IL est situé au Sud-Est du Tchad entre les latitudes $10^{\circ} 34^{\prime}$ et $11^{\circ} 03^{\prime}$ Nord et les longitudes $19^{\circ} 21^{\prime}$ et $20^{\circ} 00^{\prime}$ Est, dans la région du Salamat, deuxième grande zone d'inondation du bassin tchadien (figure 1).

Il se trouve à la limite des zones bioclimatiques soudanienne et sahélienne. Il est donc soumis à un climat tropical sec, nettement continental, avec des écarts de température, d'humidité et de précipitations assez importants (AUBRÉVILLE, 1949 ; LE HOUEROU, 1989). La saison des pluies s'étend de juin à septembre avec une moyenne annuelle de $800 \mathrm{~mm}$ (figure 2). La température moyenne annuelle est de $27^{\circ} \mathrm{C}$ et varie relativement peu au cours de l'année (DEJACE, 2002). Le relief peu contrasté permet en saison des pluies au réseau hydrographique, représenté par le Bahr Salamat et ses principaux affluents, de produire de vastes inondations annuelles qui assurent l'alimentation en eau de grandes plaines herbeuses marécageuses.

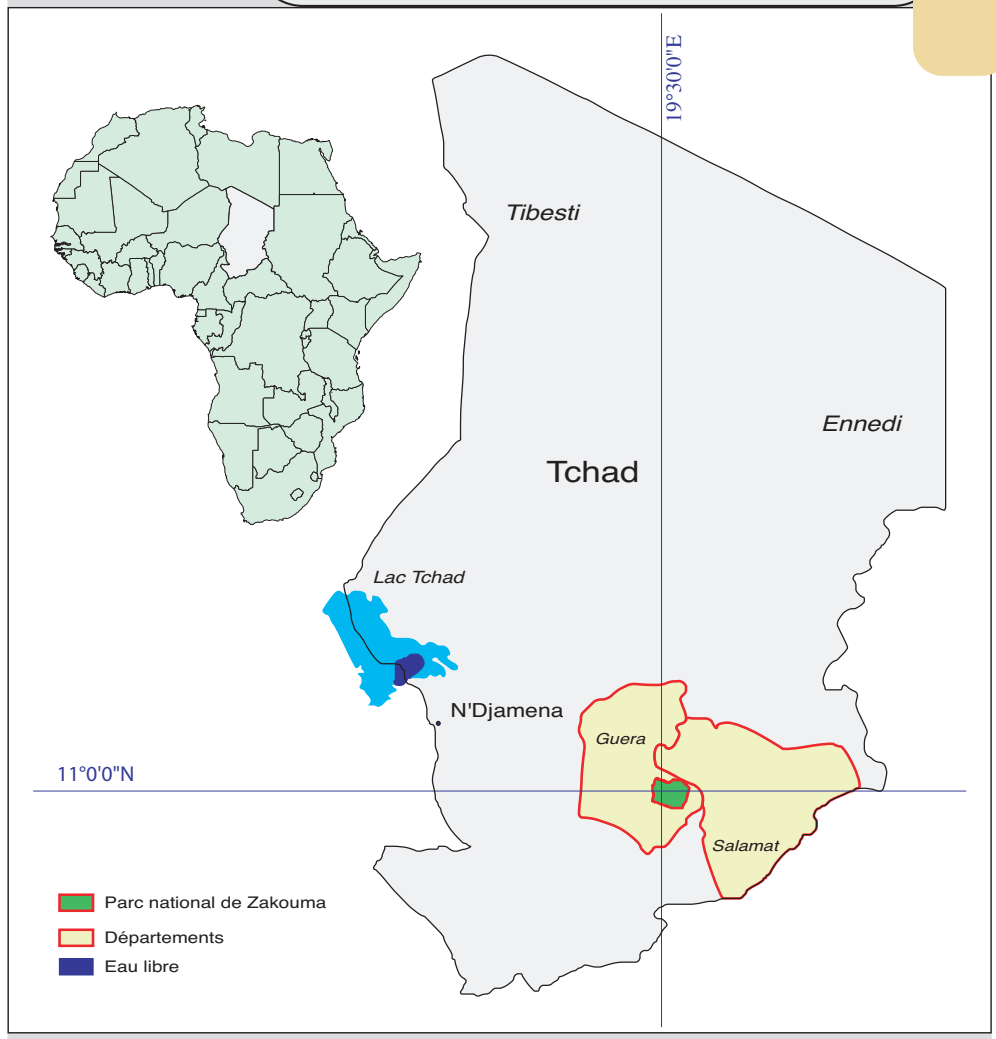

Figure 1.

Localisation du parc national de Zakouma.

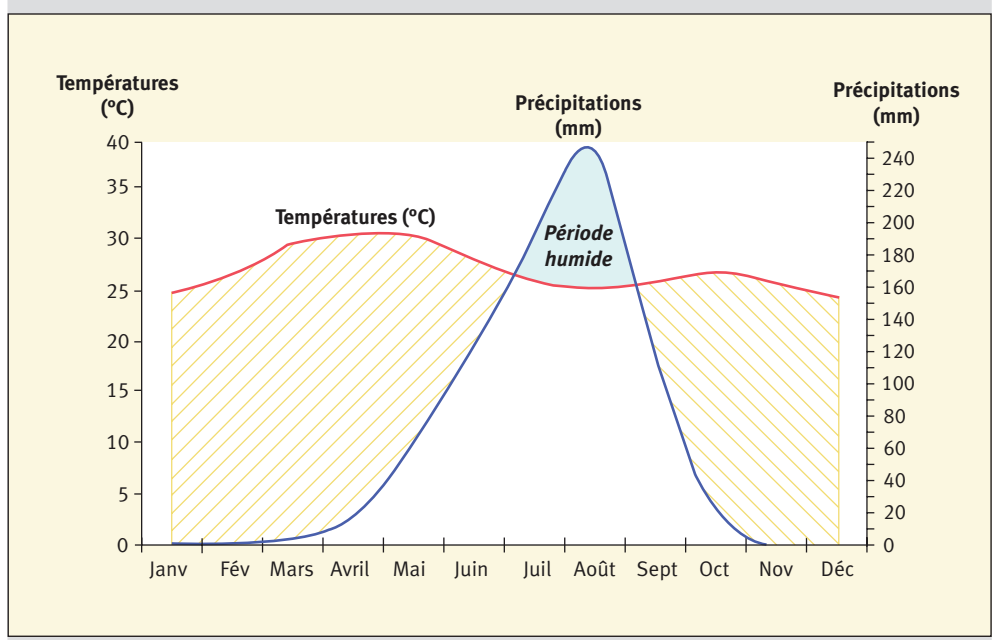

Figure 2.

Diagramme ombrothermique du parc de Zakouma présentant les précipitations mensuelles moyennes et les températures mensuelles moyennes de 1950 à 1990.

D’un point de vue morpho-pédologique, le parc de Zakouma est caractérisé par deux grandes régions : les couloirs alluviaux des Bahrs qui forment une vaste zone dépressionnaire à l'Est, et à l'Ouest un prolongement des contreforts du Massif central tchadien, représenté par de petits massifs granitiques qui surplombent un ensemble cuirassé (PIAS, BARBERY, 1965). Quant à la végétation, elle est principalement représentée par des savanes à Acacia seyal ou à Combretaceae ainsi que par de vastes étendues herbeuses marécageuses (POILECOT et al., 2009).

1 Projet Curess (Conservation et utilisation rationnelle des écosystèmes soudano-sahéliens). 


\section{Matériel et méthodes}

C'est une image Spot 5 à haute résolution spatiale, capteur multispectral XS, du 28 novembre 2004, accompagnée de 114 relevés floristiques (placettes circulaires), qui a été utilisée. Les relevés floristiques ont été exécutés en période de saison sèche (d'octobre 2004 à avril 2005) et ne concernent que les espèces ligneuses, cibles prioritaires de ces grands pachydermes à cette époque de l'année.

\section{La végétation ligneuse}

Dans cette végétation formée de mosaïques ligneuses de densité variable, des niveaux successifs de discontinuité apparaissent au fur et à mesure que l'échelle croît. De ce fait, et compte tenu de la difficulté de perception de l'homogénéité stationnelle, la planification du dispositif d'échantillonnage a été de type systématique (GODRON et al., 1968 ; GOUNOT, 1969 ; FRONTIER, 1983). À partir d'une grille dont l'unité de mesure est une maille de $1 \mathrm{~km}^{2}, 40$ transects de $1 \mathrm{~km}$ de long furent choisis et matérialisés (figure 3).

Sur chaque transect ont été positionnées trois placettes couvrant chacune $803 \mathrm{~m}^{2}$, soit au total 120 placettes, dont six ont été écartées car n'appartenant pas aux formations à Acacia seyal. C'est à l'intérieur de chaque placette, géoréférencée, qu'un inventaire de toutes les espèces ligneuses a été réalisé. Quant à l'appréciation de la prise alimentaire des éléphants, celle-ci a été caractérisée en notant les arbres déracinés, les troncs cassés ainsi que toutes les branches cassées (maîtresses et secondaires) dont le diamètre est supérieur à $2 \mathrm{~cm}$. Un indice de préférence alimentaire est calculé en référence à PETRIDES (1975) et ISHWARAN (1983) :

IP (indice de préférence) $=U / D$

Avec :

U (Taux d'utilisation) $=100 \times \frac{\text { Nombre d'arbres broutés de l'espèce }}{\text { Nombre total d'individus de l'espèce }}$

et

$D($ Potentiel disponible $)=100 \times \frac{\text { Nombre d'individus de l'espèce }}{\text { Nombre total d'individus inventoriés }}$

\section{Les images satellitaires}

La rectification des distorsions géométriques dues aux instruments de mesure est réalisée avec le logiciel Erdas Imagine ${ }^{\circledR}$. Cette opération repose sur la localisation de points invariants (huit points ont été choisis) bien répartis sur toute la zone étudiée et relevés par Gps sur le terrain (croisement de routes, croisement de pistes aéronautiques...). L'image XS est géométriquement corrigée et orthorectifiée à l'aide du modèle numérique d'élévation (Ref3D) en utilisant le référentiel spatial Utm fuseau 34 Nord (Universal Transverse Mercator). La précision ainsi obtenue est de l'ordre de $3 \mathrm{~m}$ (0,3 pixel).

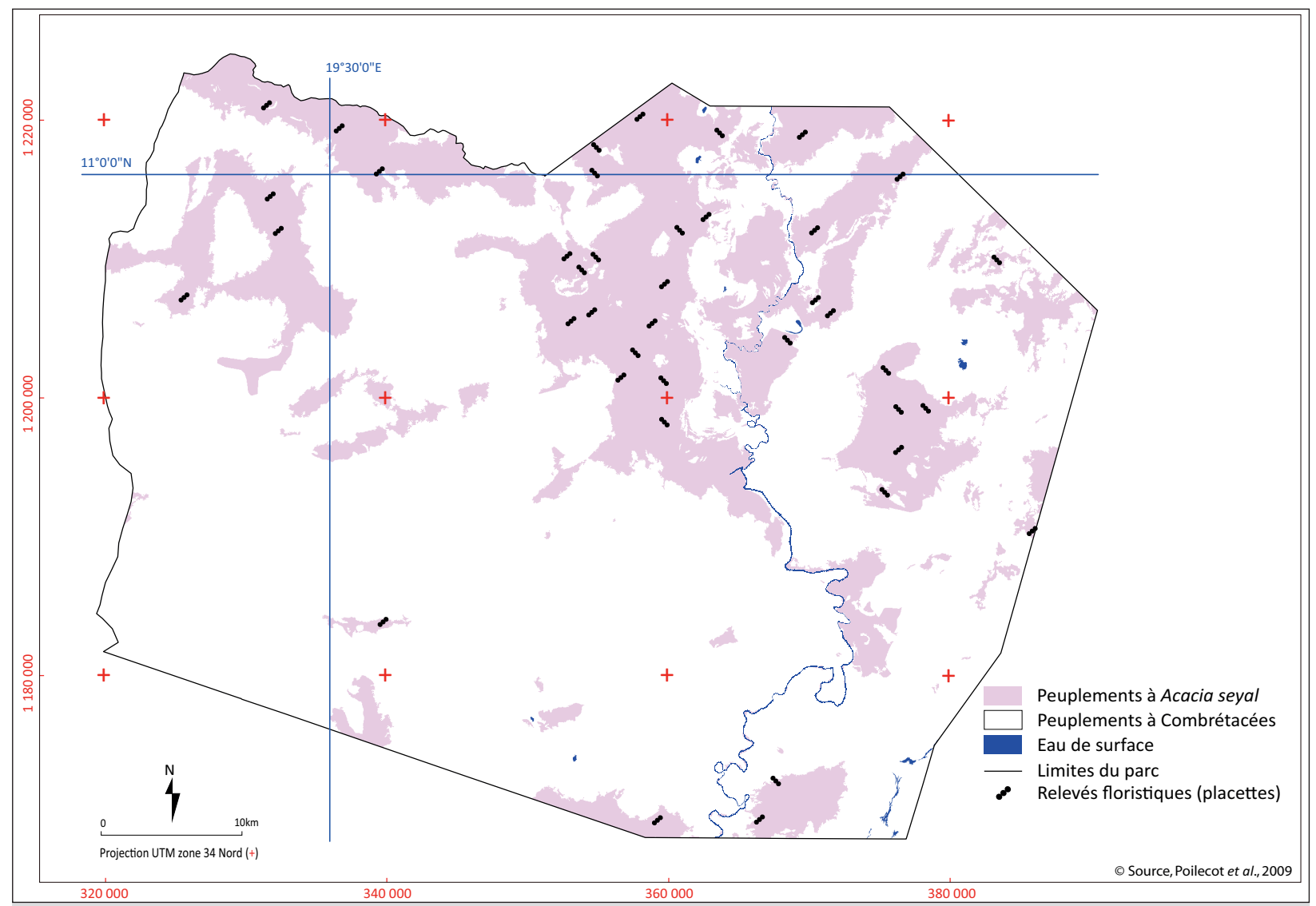

Figure 3.

Plan d'échantillonnage pour les inventaires de la végétation dans les savanes à Acacia seyal. 
Quant aux défauts radiométriques, ponctuels ou en lignes, dus essentiellement aux capteurs embarqués à bord du satellite, ils sont corrigés par une interpolation au plus proche voisin (Ppv). Ce reformatage radiométrique, réalisé sous Erdas Imagine ${ }^{\circledR}$, permet non seulement de préserver la valeur initiale du pixel, mais aussi l'élimination du lignage et la calibration relative des canaux.

Les corrections atmosphériques sont indispensables pour une comparaison multidate. Elles ont été appliquées strictement dans un souci de précision pour les traitements relatifs à la corrélation radiométrie-densité. Trois étapes sous Erdas Imagine ${ }^{\circledR}$ sont réalisées. Il s'agit du passage du compte numérique en mode luminance de chaque bande spectrale, du passage du mode luminance en mode réflectance audessus de l'atmosphère et enfin du passage du mode réflectance au-dessus de l'atmosphère en mode réflectance au-dessus du sol. Cette dernière étape est réalisée à l'aide du module Atcor ${ }^{2}$ d'Erdas Imagine ${ }^{\circledR}$ (suppression des effets atmosphériques tels que la vapeur d'eau et les particules d'aérosols qui influencent l'enregistrement du signal et de ceux d'illumination tels que les pentes à l'ombre ou au soleil qui modifient les comportements spectraux des surfaces).

\section{L’accessibilité relative des points d'eau}

L'eau étant la contrainte environnementale majeure en saison sèche, sa répartition spatiale et sa disponibilité dans le temps conditionnent le fonctionnement des déplacements des troupeaux d'éléphants. De ce fait, en cette période de l'année, les points d'eau permanents ont constitué le point de départ de cette analyse spatiale.

La zone d'étude est considérée comme un espace non homogène et non isotrope du fait que l'action du pâturage est modulée par le relief ou par les voies d'accès naturelles. Un modèle numérique d'élévation (Ref3D de Spot Image) dont la résolution est de $20 \mathrm{~m}$ a été utilisé et a permis l'application d'une fonction du déplacement-pâturage d'un troupeau d'éléphants permettant de calculer les aires de pâturage à travers une grille de coût (Cost-Grid) entre un point de départ (mare ou segment d'eau permanent) et les peuplements d'acacias environnants.

La fonction Pathdistance, disponible sous ArcInfo Gis 9.3, a été utilisée pour calculer le coût cumulé (en unité de temps) sur une surface représentant l'espace pâturé (SAÏDI, GINTZBURGER, 2013). Cette surface est une grille de pixels (Cost-Grid) définissant le coût d'impédance ou de déplacement planimétrique à travers chaque cellule selon une vitesse (à plat) de 1,2 s/m, soit une vitesse de $3 \mathrm{~km} / \mathrm{h}$ (mesure appréciée sur le terrain en situation de déplacementpâturage). Cette vitesse est variable en fonction de la pente et de la topographie, deux contraintes prises en compte dans le calcul des aires de pâturage autour du point d'eau de départ

2 Le module Atcor est développé au Centre aérospatial allemand de Wessling (RICHTER, 1996, 1997). Il intègre une base de données atmosphériques comprenant une large gamme de calculs du transfert radiatif adaptée aux différentes conditions météorologiques et d'angles solaires (zénith, azimut), sur la base du code Modtran-4 (BERK et al., 2003). 


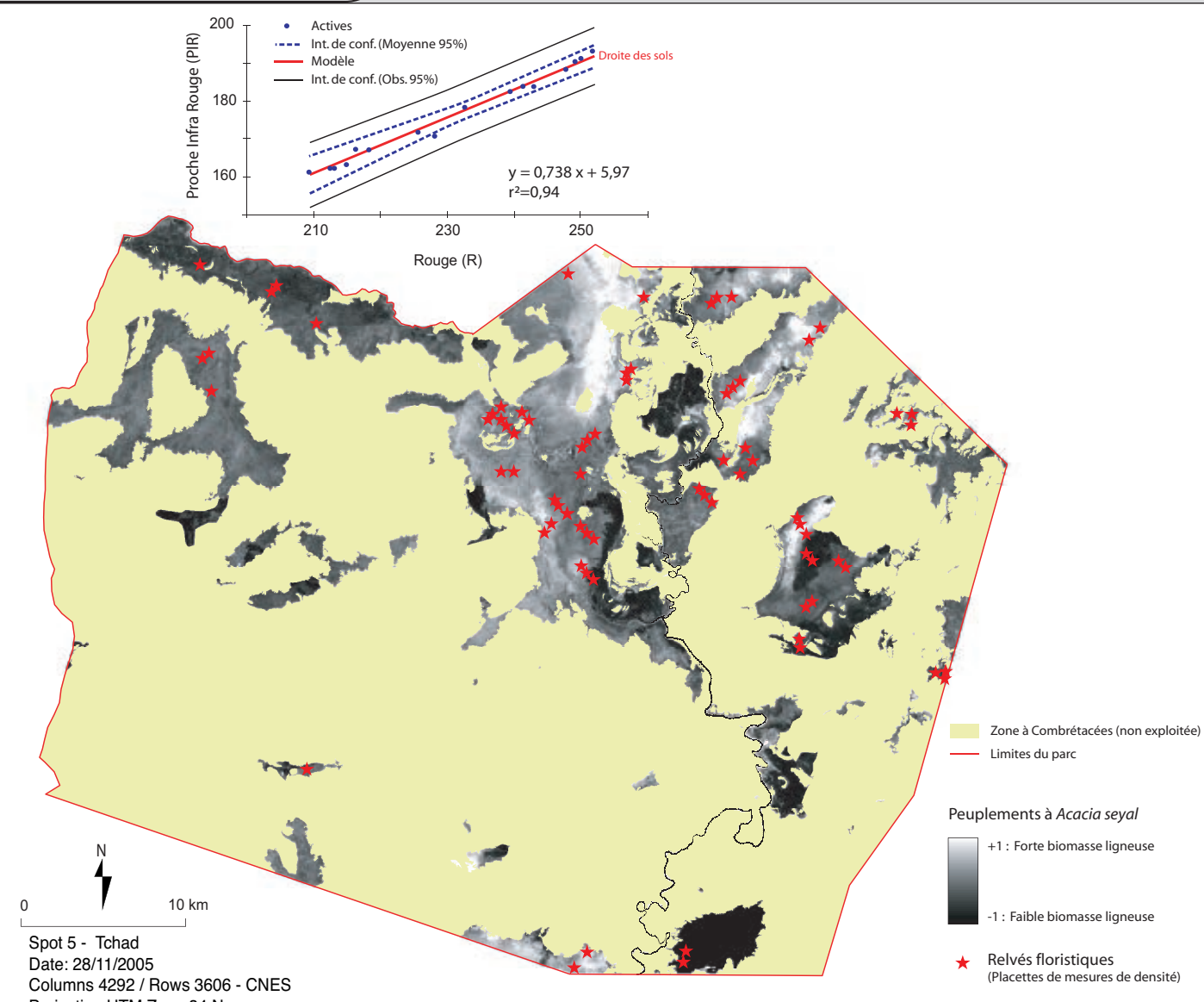

Projection UTM Zone $34 \mathrm{~N}$

Datum: WGS 84

Figure 4.

L'indice perpendiculaire de végétation.

points des surfaces de ces rectangles $\left(x_{i}-x_{p 3}\right)$ et $\left(y_{i}-y_{p 3}\right)$, appelée la somme des produits des écarts au modèle. Cet indice prend en compte le poids de la réflectance des sols nus par rapport à la réflectance de la végétation. Son calcul donne une meilleure discrimination de la végétation en zone sèche. Il est exprimé par l'équation suivante :

$P V I=\frac{P I R-(a R+b)}{\sqrt{a^{2}+1}}$

Avec $a$ et $b$ qui sont respectivement la pente et l'ordonnée à l'origine. Un PVI positif signifie que le pixel est recouvert par la végétation, un PVI nul correspond aux pixels de sol nu et un PVI négatif est obtenu pour les surfaces en eau de très faible teneur minérale ou chlorophyllienne.

\section{La biomasse ligneuse}

Elle peut être estimée à partir de la mesure de la surface de la couronne qui en est la projection verticale. Cependant, dans le cas d'une imbrication des différentes couronnes des individus de la station (forêts denses et moyennement denses), la notion de projection verticale surestime fortement cette biomasse. Afin d'éviter ce biais, nous avons privilégié plutôt la prise en compte de la notion de densité pour cartographier la distribution géographique des formations à Acacia seyal.

L'utilisation de la notion de densité plutôt que celle de couronne est justifiée par la qualité de la relation densité des ligneux observés/surface de leur couronne $\left(r^{2}=78 \%\right.$; figure 5). De ce fait, la mise en corrélation des mesures de densités avec les valeurs moyennes du PVI de l'image XS a été privilégiée.

L'équation de la relation densité ligneuse/PVI moyen a permis de cartographier une estimation quantitative de la ressource ligneuse des formations à Acacia seyal (figure 6). Celleci est traduite par une nouvelle zonation de la carte du peuplement d'Acacia seyal réalisée par POILECOT et al. (2009).

Les valeurs moyennes du PVI sont calculées sur des zones de $20 \times 20$ pixels, soit une surface de 4 ha, et cela pour compenser l'incertitude de localisation de nos placettes. Cette surface de 4 ha constitue un point important dans la classification car plus elle est grande et homogène, meilleure est la segmentation des classes de densité. L'erreur standard entre les valeurs des densités mesurées sur le terrain et celles calculées par la régression est de l'ordre de 23 arbres par hectare. 


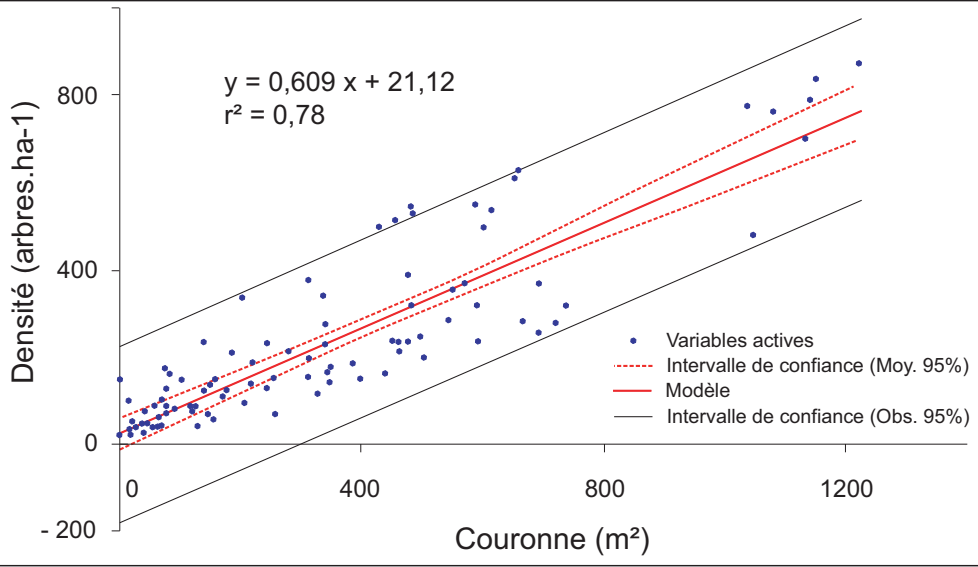

Figure 5.

Relation de la densité de végétation avec la surface de la couronne.

\section{La perception du peuplement d'Acacia seyal}

En saison sèche, la stratégie alimentaire des éléphants s'oriente vers les peuplements ligneux permettant de bénéficier d'un contenu en protéines plus élevé (FIELD, 1971 ; JACHMANN, BELL, 1985).

La prise en compte de la notion de valeur pastorale (VP) dans ce travail (indicateur plutôt destiné à l'élevage ovin et bovin) montre que la dynamique spatiale des éléphants semble s'affranchir de cet indicateur. La fréquentation animale est de l'ordre de $76 \%$ de présence sur des espaces de faible valeur pastorale contre $24 \%$ sur ceux plutôt considérés de bonne qualité fourragère (figure 6).

À l'origine, VP caractérise la valeur du tapis végétal de la station (TCHABI, 1996). Il prend en compte l'abondance relative des espèces ligneuses (mesurée par leur contribution spécifique : $\mathrm{CS}_{(i)}$ ) et

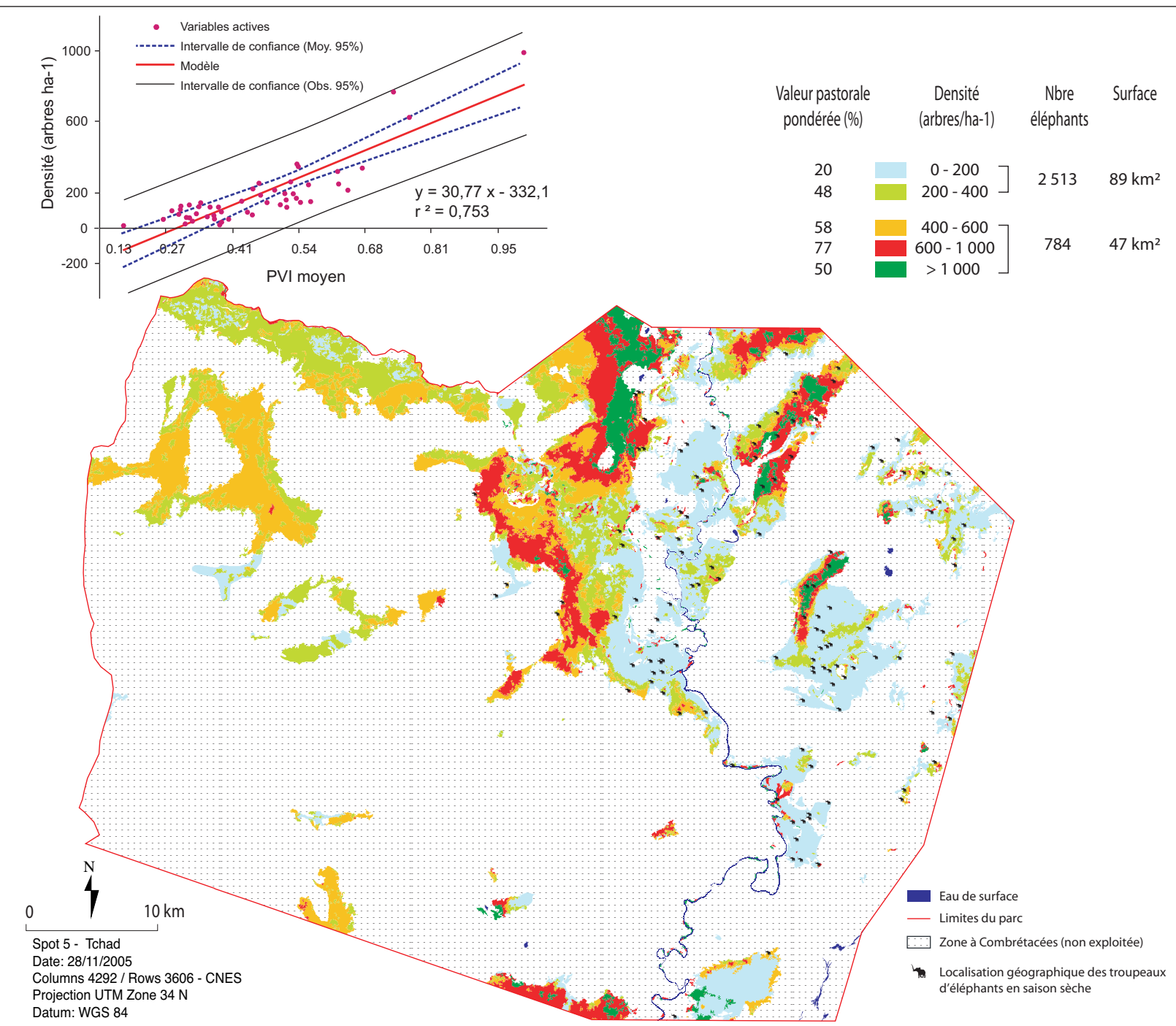

Figure 6.

Densité des ligneux au sein du peuplement à Acacia seyal. 
leur qualité (mesurée par l'indice spécifique : Is ${ }_{(i)}$ qui varie de 1 à $5 ; 5=$ excellent fourrage ; $4=$ très bon fourrage ; $3=$ bon fourrage ; 2 = fourrage moyen ; 1 = fourrage médiocre) (DAGET, POISSONET, 1969 ; CESAR, COULIBALY, 1993 ; BOUDET, 1991), selon la formule suivante :

$V P=\frac{C S(i) \cdot I S(i)}{5}$

Pour caractériser la station, il faut introduire le degré de recouvrement global de la végétation R\% (DAGET, 1995) :

$V P($ station $)=V P \cdot \frac{R}{100}$

Lorsque la station dont il faut évaluer la valeur est hétérogène, chaque élément est caractérisé séparément par sa VP (station) et les différentes valeurs sont combinées et pondérées par l'importance de chaque élément ; ainsi, pour $n$ éléments d'aire $S_{(i)}$, la valeur d'ensemble est :

$V P($ Ensemble $)=\frac{\Sigma_{\mathrm{n}} V P(i) \cdot S(i)}{\Sigma_{\mathrm{n}} S(i)}$
La figure 6 montre que la dynamique spatiale des éléphants dans cette partie du parc au cours des saisons sèches de 2002, 2005, 2006 et 2008 reste majoritairement localisée dans des espaces où la densité ligneuse est inférieure à 400 arbres par hectare. Cette figure montre également que plus de 2500 éléphants ont fréquenté ces espaces (soit $76 \%$ ), contre 784 pour les zones les plus denses (soit $24 \%$ ). Ces $76 \%$ de présence animale, observées en saison sèche, manifestent l'intérêt des éléphants pour des espaces jugés plutôt de faible qualité fourragère par les pastoralistes.

Ce seuil de 400 arbres par hectare est également observé au travers de l'ordination des 114 placettes analysées selon les critères « impact sur la végétation ligneuse » et « densité ligneuse ». Trois groupes distincts de placettes se sont dégagés (figure 7) :

- un groupe de 71 placettes caractérisé par des densités inférieures à 400 arbres par hectare ;

- un groupe de 14 placettes restreint plutôt aux densités supérieures à 400 arbres par hectare ;

- un groupe qui renferme 29 placettes dans lequel aucun impact sur la végétation ligneuse n'a été observé (ce groupe a été écarté).
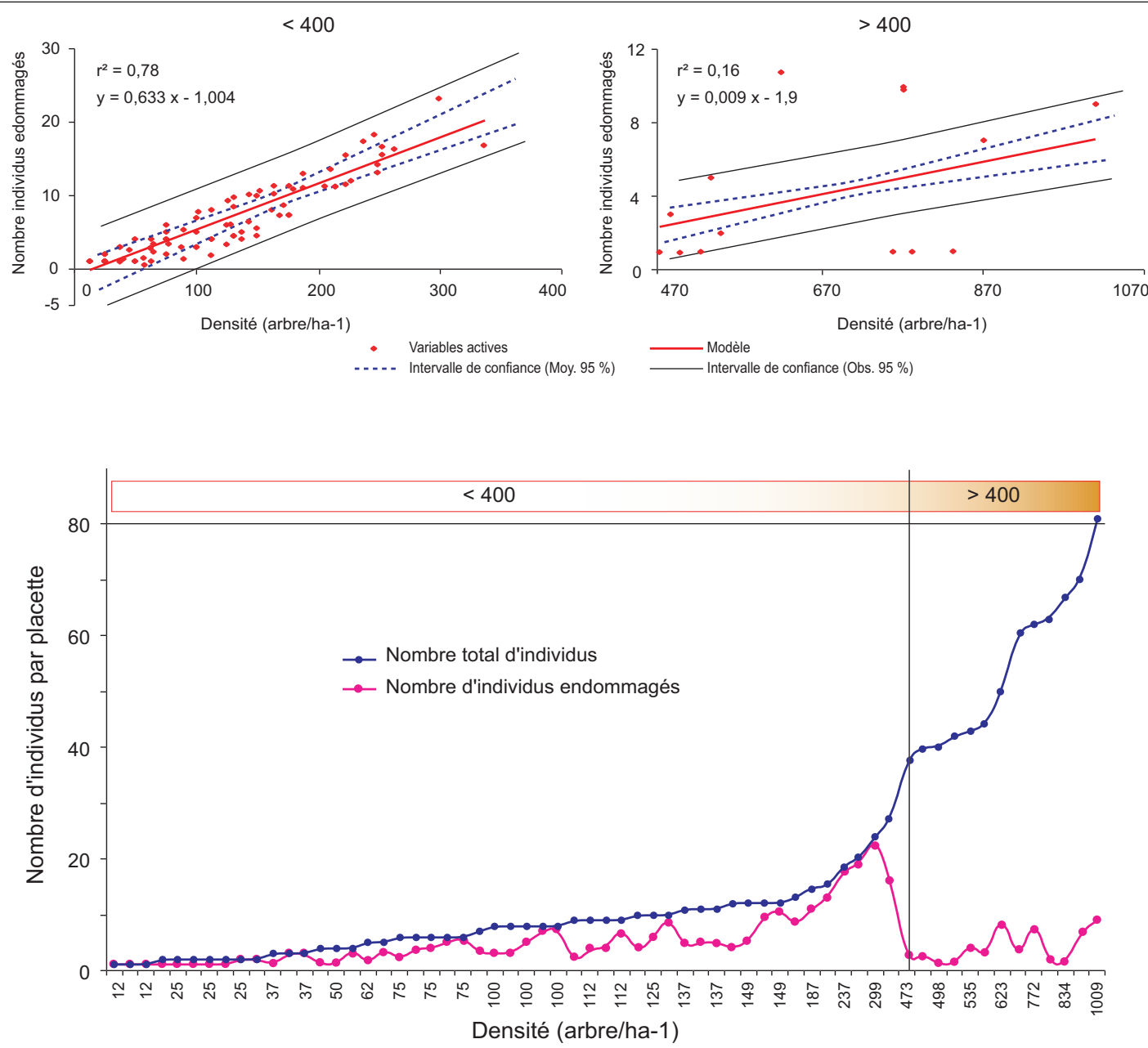

Figure 7.

Relation de la densité ligneuse avec la prise alimentaire chez les éléphants dans les peuplements d’Acacia seyal. 
Ce constat est éclairé par la relation ci-dessous entre la densité des ligneux et l'intensité de la prise alimentaire (figure 7), établie sur la base de 85 relevés où l'impact des éléphants sur la végétation ligneuse a été significatif.

Cette perception de l'espace selon un seuil de densité ligneuse à partir duquel ces herbivores limitent leur accès en saison sèche aux peuplements d'Acacia seyal ne semble donc pas liée à la valeur alimentaire de la végétation telle qu'elle est perçue par les pastoralistes. En effet, l'ensemble des placettes exécutées dans ces espaces ouverts présentent une valeur fourragère ne dépassant guère 47 \% (figure 6), ce qui correspond à une végétation de faible qualité fourragère pour le bétail (DAGET, GODRON, 1995). Selon ces auteurs, une végétation arbustive tropicale de bonne qualité posséderait plutôt une valeur supérieure à $65 \%$.

L'analyse de la prise alimentaire de ces pachydermes montre également que Acacia polyacantha et Balanites aegyptiaca apparaissent comme des espèces très appréciées (tableau I). Ce constat reste néanmoins à confirmer car, sur les 1155 arbres inventoriés, Acacia polyacantha et Balanites aegyptiaca ne représentent respectivement que $0,5 \%$ et $1 \%$ du nombre total des individus observés. Suivent Acacia nilotica, Cadaba farinosa, Combretum glutinosum, Feretia apodanthera, Acacia sieberiana, Lannea humilis et Piliostigma reticulatum qui traduisent des taux relativement importants. Ces espèces, placées dans un environnement à flore diversifiée et abondante, restent peu consommées par le bétail car ce fourrage ne lui est guère profitable.
Finalement, malgré sa forte abondance (63\% du nombre total des individus observés) et son profil alimentaire intéressant (arbre fourrager important), la contribution d'Acacia seyal apparaît dans le régime alimentaire des éléphants comme marginale. Cette espèce dominante se trouve parmi celles qui ont enregistré les plus faibles valeurs de l'indice de préférence. Ces grands herbivores semblent plutôt avoir une préférence non seulement pour les espaces ouverts (figure 6), mais aussi pour leur diversité spécifique qui se traduit par la présence d'espèces telles que Acacia polyacantha, Balanites aegyptiaca, Acacia nilotica, Cadaba farinosa, Combretum glutinosum, Feretia apodanthera et Acacia sieberiana, nettement moins abondantes que Acacia seyal (tableau I).

\section{Une stratégie de pâturage de " proximité "}

En saison sèche, la quête alimentaire des éléphants est considérablement influencée par la permanence des points d'eau, considérés comme un facteur déterminant dans la perception de l'espace pâturé. En cette période de l'année, ces grands herbivores ont développé des mécanismes qui leur permettent d'éviter, de tolérer ou de contrôler l'excès de chaleur lors des phases de pâturage. Outre l'importante surface de leurs pieds pour faciliter leur déplacement sur les sols sableux et leurs grandes oreilles pour dissiper la chaleur en faisant office d'éventail, ils adoptent une stratégie de pâturage de «proximité » : plus les savanes

Tableau I.

Intensité des prélèvements dans les peuplements ligneux des savanes à Acacia seyal

\begin{tabular}{|c|c|c|c|c|c|}
\hline Espèce & $\begin{array}{l}\text { Nombre } \\
\text { d'arbres } \\
\text { observés }\end{array}$ & $\begin{array}{l}\text { Nombre } \\
\text { d'arbres } \\
\text { impactés }\end{array}$ & $\begin{array}{c}\text { Taux } \\
\text { d'utilisation (U) }\end{array}$ & $\begin{array}{c}\text { Potentiel } \\
\text { disponible (D) }\end{array}$ & $\begin{array}{c}\text { Indice de } \\
\text { préférence (U/D) }\end{array}$ \\
\hline Acacia polyacantha & 6 & 2 & 33,3 & 0,5 & 64,2 \\
\hline Balanites aegyptiaca & 11 & 3 & 27,3 & 1 & 28,6 \\
\hline Acacia nilotica & 22 & 8 & 36,4 & 1,9 & 19,1 \\
\hline Cadaba farinosa & 18 & 5 & 27,8 & 1,6 & 17,8 \\
\hline Combretum glutinosum & 12 & 2 & 16,7 & 1 & 16 \\
\hline Feretia apodanthera & 20 & 5 & 25 & 1,7 & 14,4 \\
\hline Acacia sieberiana & 37 & 17 & 45,9 & 3,2 & 14,3 \\
\hline Lannea humilis & 18 & 2 & 11,1 & 1,6 & 7,1 \\
\hline Piliostigma reticulatum & 70 & 26 & 37,1 & 6,1 & 6,1 \\
\hline Ziziphus mauritiana & 16 & 1 & 6,3 & 1,4 & 4,5 \\
\hline Dichrostachys cinerea & 51 & 9 & 17,6 & 4,4 & 4 \\
\hline Capparis tomentosa & 30 & 2 & 6,7 & 2,6 & 2,6 \\
\hline Combretum aculeatum & 40 & 2 & 5 & 3,5 & 1,4 \\
\hline Anogeissus leiocarpa & 43 & 1 & 2,3 & 3,7 & 0,6 \\
\hline Acacia seyal & 735 & 264 & 35,9 & 63,6 & 0,6 \\
\hline Mitragyna inermis & 14 & 0 & 0 & 1,2 & 0 \\
\hline Boscia senegalenis & 7 & 0 & 0 & 0,6 & 0 \\
\hline Flueggea virosa & 3 & 0 & 0 & 0,3 & 0 \\
\hline Bauhinia rufescens & 1 & 0 & 0 & 0,1 & 0 \\
\hline Tamarindus indica & 1 & 0 & 0 & 0,1 & 0 \\
\hline
\end{tabular}


sont proches du point d'eau et plus celles-ci sont exploitées. Cette proximité à l'eau limite dans le temps leurs déplacements et les contraint à se situer entre 3 à 6 heures de marche par rapport au point d'eau le plus proche, soit une distance maximale tolérée entre 0 et $9 \mathrm{~km}$, allant jusqu'à 18 km (figure 8). IPAVEC (2007) évoque une distance de $9 \mathrm{~km}$ quand la ressource en eau devient rare. Cette stratégie de pâturage en cette saison de l'année permet de compenser les dépenses énergétiques liées aux déplacements de retour à l'eau (OWEN-SMITH, 1988).

POILECOT (2010) retrouve les remarques de MACKIE (2002) et de FAY et al. (2005, 2006), qui indiquent qu'en saison sèche la distribution des éléphants dans la partie Est du parc de Zakouma demeure essentiellement liée à la permanence du réseau hydrographique.

\section{Discussion et conclusion}

En saison sèche, les quelques mares résiduelles du Bhar Salamat « attirent » les éléphants du parc de Zakouma vers les savanes d'Acacia seyal. Cette dépendance à l'eau oriente leurs déplacements et induit une concentration inéluctable en ces lieux qui influencent considérablement leur quête alimentaire. Simultanément, les sites présentant un recouvrement arbustif de moins de 400 arbres à l'hectare et offrant, dès les premières pluies, de vigoureuses repousses végétales sont sélectivement recherchés. En règle générale, les peuplements à forte densité deviennent des barrières physiques entravant les déplacements des éléphants et réduisant ainsi l'utilisation de ces espaces.

Au sein de ces peuplements riches en Acacia seyal, la contrainte que représente la disponibilité en eau incite les éléphants du parc à "s'organiser » pour satisfaire leurs besoins nutritionnels en fonction de la proximité géographique de la ressource hydrique. Cette proximité est prépondérante vis-à-vis des divers schémas d'utilisation de cet écosystème de savane. La majorité des troupeaux observés se localisent à moins de $9 \mathrm{~km}$ de la ressource en eau, rarement au-delà de $18 \mathrm{~km}$. Ces résultats sont compatibles avec les observations rapportées ailleurs en Afrique australe (CONYBEARE, 1991 ; STOKKE, DU TOIT, 2002 ; REDFERN et al., 2003 ; LEGGET, 2006 ; CHAMAILLÉ-JAMMES et al., 2006) et en Afrique orientale (AYENI, 1979 ; GALANTI et al., 2006).

Les espèces préférentiellement consommées par les éléphants ont toutes été observées dans des stations à recouvrement arbustif inférieur à 400 arbres à l'hectare. Le fait de privilégier un léger recouvrement arboré en se tenant à proximité géographique de l'eau permet aux éléphants en saison sèche d'accéder plus facilement à des jeunes plants et plus particulièrement à leurs racines (CHRISTENSON, 1976 in SPINAGE, 1985). Ces repousses présentent de fortes teneurs en nutriments et sont très recherchées en période critique de l'année (OWEN-SMITH, 1988).

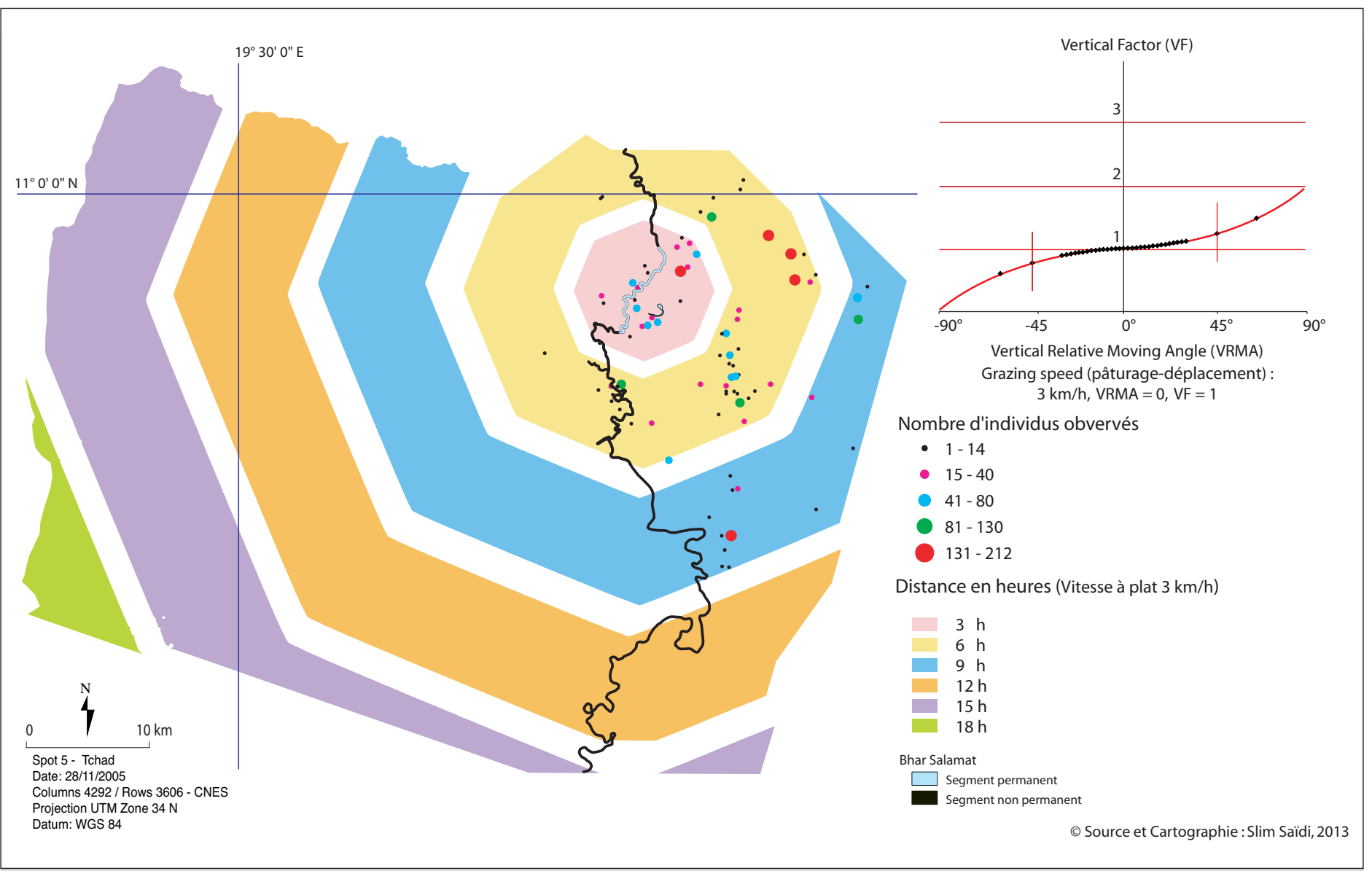

Figure 8.

Les aires de pâturage des éléphants autour d'un point d'eau permanent. 
Dix espèces végétales sont régulièrement consommées dans la partie orientale du parc. Il s'agit d'Acacia polyacantha, Balanites aegyptiaca qui présentent un niveau de consommation élevé d'Acacia nilotica, Cadaba farinosa, Combretum glutinosum, Feretia apodanthera et Acacia sieberiana qui enregistrent plutôt un niveau de consommation moyen et, enfin, Lannea humilis, Piliostigma reticulatum, Ziziphus mauritiana, Dichrostachys cinerea, Capparis tomentosa qui enregistrent un niveau de consommation faible. Quant à Combretum aculeatum et Anogeissus leiocarpa, ces deux espèces montrent un niveau de consommation très faible. Il en est de même pour Acacia seyal qui, malgré son abondance et son excellent profil fourrager, traduit une contribution médiocre dans la quête alimentaire des éléphants.

Au terme de ce travail, il apparaît que, dans cette partie orientale du parc de Zakouma, la préférence alimentaire des éléphants dépend conjointement de la proximité de la ressource en eau et des zones faiblement arborées, plutôt propices à l'installation de jeunes plants. Ce constat demeure conforme aux observations faites par OWENSMITH (1988) et JACHMANN et CROES (1991), qui montrent que ce sont les individus jeunes adultes pouvant évoluer dans des espaces plutôt ouverts qui sont davantage recherchés et qui ne présentent pas obligatoirement des profils fourragers intéressants.

\section{Références bibliographiques}

AUBRÉVILLE A., 1949. Climats, forêts et désertification de l'Afrique tropicale. Paris, France, Société d'éditions géographiques, maritimes et coloniales, $351 \mathrm{p}$.

AYENI J. S. O., 1979. Distribution of wildlife in relation to the waterholes in Tsavo National Park (East), Kenya. In: Wildlife management in savannah woodland. Recent progress in African studies. Londres, Royaume-Uni, Taylor and Francis, p. 67-84.

BERK A., ANDERSON G. P., ACHARYA P. K., HOKE M. L., CHETWYND J. H., BERNSTEIN L. S., SHETTLE E. P., MATTHEW M. W., ADLER-GOLDEN S. M., 2003. MODTRAN4 Version 3 Revision 1 User's Manual. Hanscom, MA, États-Unis, Air Force Research Laboratory.

BOUDET G., 1991. Manuel sur les pâturages tropicaux et les cultures fourragères. Paris, France, lemvt, Ministère de la Coopération et du Développement, coll. Précis d'élevage, 226 p.

CALENGE C., MAILLARD D., GAILLARD J., MERLOT L., PELTIER R., 2002. Elephant damage to trees of wooded savannas in Zakouma National Park, Chad. Journal of Tropical Ecology, 18: 599-614.

CESAR J., COULIBALY A., 1993. Conséquences de l'accroissement démographique sur la qualité de la jachère dans le nord de la Côte d’Ivoire. In : Serpantié G. (éd.). La jachère en Afrique de l'Ouest. Paris, France, Orstom, coll. Colloques et séminaires, p. 415-433.
CHAMAILLÉ-JAMMES S., FRITZ H., MURINDAGOMO F., 2006. Spatial patterns of the NDVI-rainfall relationship at the seasonal and interannual time scales in an African savanna. International Journal of Remote Sensing, 27: 5185-5200.

CONYBEARE A. M., 1991. Elephant occupancy and vegetation change in relation to artificial water points in a Kalahari sand area of Hwange National Park. Degree of Doctor of Philosophy, Department of Biological Sciences, University of Zimbabwe, $420 \mathrm{p}$.

DAGET PH., 1995. Valeur alimentaire de la végétation. In : Daget PH., Godron M. (coord.). Pastoralisme. Troupeaux, espaces et sociétés. Paris, France, Hatier, p. 241-246.

DAGET PH., POISSONET J., 1969. Analyse phytologique des prairies. Applications agronomiques. Montpellier, France, Cnrs-Cepe, doc n 48, 67 p.

DARVISHZADEH R., SKIDMORE A., ATZBERGER C., VAN WIEREN S., 2008. Estimation of vegetation LAI from hyperspectral reflectance data: Effects of soil type and plant architecture. International Journal of Apply Earth Observation and Geoinformation, 10: 358-373.

DEJACE P., 2002. Zakouma. N’Djaména, Tchad, Ministère de l'Environnement et de l'Eau, Commission Européenne, 248 p.

FAY M., DOLMIA N. M., BOULANODJI E., POILECOT P., NDONINGA A., GUGGEMOS C., 2005. Comptage aérien total de la grande faune du Parc national de Zakouma. N’Djaména, Tchad, Ministère de l'Environnement et de l'Eau, Dcfap, Curess.

FAY M., NGAKOUTOU E. B., TALOUA N., POILECOT P., NDONINGA A., 2006. Dénombrement aérien total des grands mammifères et de l'autruche du Parc national de Zakouma, Tchad. N’Djaména, Tchad, Ministère de l'Environnement et de l'Eau, Dcfap, Curess.

FIELD C. R., 1971. Elephant ecology in the Queen Elisabeth National Park, Uganda. East African Wildlife Journal, 9: 99-123.

FRONTIER S., 1983. Stratégies d'échantillonnage en écologie. Paris, France, Masson, 494 p.

GALANTI V., PREATONI D., WAUTERS L. A., TOSI G., 2006. Space and habitat use of the African elephant in the Tarangire-Manyara ecosystem, Tanzania: implications for conservation. Mammalian Biology, 71: 99-114.

GINTZBURGER G., SAIIDI S., SOTI V., 2005. Rangelands of the Ravnina region in the Karakum desert of Turkmenistan: Current condition and utilization. In: Desertification and regeneration: modeling the impact of market reform on Central Asian rangeland, 122 p. ICARDA reprint, www.icarda.org, Sept. 2009. Available at: http://www.icarda.org/cac/sacac.asp.

GODRON M., DAGET PH., EMBERGER L., LE FLOC'H E., LONG G., POISSONET J., SAUVAGE CH., WACQUANT J.-P., 1968. Code pour le relevé méthodique de la végétation et du milieu. Paris, France, Cnrs, 292 p.

GOUNOT M., 1969. Méthodes d'étude quantitative de la végétation. Paris, France, Masson, 314 p. 
IPAVEC A., 2007. Caractéristiques et déterminants de la distribution spatio-temporelle de la population d'éléphants du parc régional du W, Afrique de l'Ouest. Thèse, Université de Rennes, France, 231 p.

ISHWARAN N., 1983. Elephant and woody plant relationship in Gal Oya, Sri Lanka. Biological Conservation, 26: 255-270.

JACHMANN H., BELL R. H. V., 1985. Utilization by elephants of the Brachystegia woodlands of the Kasungu National Park, Malawi. African Journal of Ecology, 23: 245-258.

JACHMANN H., CROES T., 1991. Effects of browsing by elephants on the Combretum/Terminalia woodland at the Nazinga Game Ranch, Burkina Faso, West Africa. Biological Conservation, $57:$ 13-24.

LE HOUEROU H.-N., 1989. Classification écoclimatique des zones arides du Nord de l'Afrique. Ecologia Mediterranea, 15 (3-4) : 95-144.

LEGGETT K., 2006. Effect of artificial water points on the movement and behaviour of desert-dwelling elephants of north-western Namibia. Pachyderm, 40: 24-34.

MACKIE C., 2002. Aerial census of elephants and other large herbivores in the Sebunge region, Zimbabwe. Harare, Zimbabwe, Dnpwlm/Wwf.

MAIRE M., 2000. Impact actuel des éléphants sur la savane à Acacia seyal : Parc national de Zakouma (Tchad). Montpellier, France, Fif-Engref, Commission européenne.

OTTERMAN J., 1996. Desert-scrub as the cause of reduced reflectance in protected vs. impacted sandy arid areas. International Journal of Remote Sensing, 17: 615-619.

OWEN-SMITH R. N., 1988. Megaherbivores. The influence of very large body size on ecology. Cambridge, Royaume-Uni, Cambridge University Press, 369 p.

PETRIDES G. A., 1975. Principal foods versus preferred foods and their relations to stocking rate and range condition. Biology Conservation, 7: 161-169.

PIAS J., BARBERY J., 1965. Notice explicative : cartes pédologiques de reconnaissance au 1/200 000. Feuilles du Lac IroDjouna. Fort-Lamy, Tchad, Centre Orstom (Ird).

PIAS J., POISOT P., 1964. Carte de reconnaissance pédologique au 1/200 000 et notice explicative du Tchad. Orstom (Ird), $150 \mathrm{p}$.

POILECOT P., 2010. La population d'éléphants du parc national de Zakouma, Tchad. Bois et Forêts des Tropiques, 303 (1) : 83-91.

POILECOT P., BOULANODJI E., TALOUA N., DJIMET B., NGUI T., SINGA J., 2007. Parc national de Zakouma : des éléphants et des arbres. Bois et Forêts des Tropiques, 291 (1) : 13-24.

POILECOT P., SAIIDI S., N'GAKOUTOU E. B., 2009. Phytogéographie du Parc national de Zakouma (Sud-Est du Tchad). Sécheresse, 20 (3) : 286-295.
REDFERN J. V., GRANT R. C., BIGGS H. C., GETZ W. M., 2003. Surface-water constraints on herbivore foraging in the Kruger National Park, South Africa. Ecology, 84: 2092-2017. RICHARDSON A. J., WIEGAND C. L., 1977. Distinguishing vegetation from soil background information. Photogrammetric Engineering and Remote Sensing, 43: 1541-1552.

RICHTER R., 1995. Atmospheric/Topographic Correction for Satellite Imagery. DLR report DLR-IB 565-01/05. Wessling, Allemagne, German Aerospace Center, 216 p.

RICHTER R., 1996. A spatially adaptive fast atmospheric correction algorithm. International Journal of Remote Sensing, 17 (6): 1201-1214.

RICHTER R., 1997. Correction of atmospheric and topographic effects for high spatial resolution satellite imagery. International Journal of Remote Sensing, 18 (5): 1099-1111.

SAÏDI S., GINTZBURGER G., 2013. A spatial desertification indicator for Mediterranean arid rangelands: a case study in Algeria. The Rangeland Journal, 35 (1): 47-62.

SPINAGE C. A., 1985. The elephants of Burkina-Faso, West Africa. Pachyderm, 5: 2-5.

STOKKE S., DU TOIT J. T., 2002. Sexual segregation in habitat use by elephants in Chobe National Park, Botswana. African Journal of Ecology, 40: 360-371.

TCHABI V., 1996. Présentation d'une formule validée établissant la relation entre les valeurs pastorales des espèces fourragères et les capacités de charge dans une réserve de faune soudanienne, Nature et Faune, 12 (4) : 30-48. 